\title{
Electronic transport through a quantum wire coupled to a quantum dot
}

\author{
Yu-Liang Liu and T. K. Ng \\ Department of Physics, Hong Kong University of Science and Technology, Clear Water Bay, Kowloon, Hong Kong, \\ People's Republic of China
}

(Received 7 June 1999)

\begin{abstract}
In this paper we re-examine the problem of electronic transports through a system consisting of a quantum dot which has well-defined discrete energy levels connected to an infinite quantum wire, using the bosonization method and phase-shift representation. We show that all previously known results can be obtained through our method in a very simple way. Furthermore, the evolution of the system from ultraviolet to infrared critical fixed points appears naturally in our method.
\end{abstract}

\section{INTRODUCTION}

Transport properties of a quantum dot connected to two reservoirs (leads) have been extensively studied ${ }^{1-9}$ recently. For a large quantum dot where energy levels are approximately continuous, the system shows Coulomb blockade behavior due to static Coulomb interaction between electrons in the dot, and its conductance is quantized. The situation is quite different for a very small quantum dot which has wellseparated energy levels. If the number of electrons in the dot is odd, which can be controlled by external gate voltages, the system shows Kondo-like low energy behavior. This property was confirmed experimentally ${ }^{10,11}$ in a system corresponding to quantum dot coupled to two leads by tunneling barriers.

In this paper, we consider another interesting situation where we replace the two reservoirs (leads) by an infinite quantum wire. For a very small quantum dot with welldefined energy levels, the tunneling conductance is determined by the level which is closest to the Fermi level of the quantum wire (assuming other levels below it are occupied by even electrons and the levels above are unoccupied). By combining the bosonization method with a phase shift representation proposed by one of us, we rederive a number of known results for this problem in this paper, including (i) the zero temperature I-V characteristic with $I \sim V_{g}^{2 / g-1}$ in the two limit cases, $\epsilon_{0} \gg \Gamma$ and $\epsilon_{0} \ll-\Gamma$, where $V_{g}$ is an external voltage, $g$ is a dimensionless coupling constant of conduction electrons, $\Gamma$ is the tunneling width between the quantum wire and dot, and $\epsilon_{0}$ is the level energy relative to the Fermi levels of the two quantum wires, and (ii) in the case $\epsilon_{0} \sim 0$, the tunneling current is proportional to the external voltage $V_{g}$. Moreover, we demonstrate that even though the tunneling current has the same low energy behavior in both cases of $\epsilon_{0} \gg \Gamma$ and $\epsilon_{0} \ll-\Gamma$, the spin susceptibility of the local electron in the quantum dot in the two cases are completely different. In the former case where the level $\epsilon_{0}$ is essentially unoccupied, the local electron level only provides a barrierlike potential scattering for conduction electrons, and its spin susceptibility is zero. In the latter case, the level $\epsilon_{0}$ is singleoccupied and the local electron has a Kondo-like exchange interaction with the conduction electrons. Its spin susceptibility shows generally low energy power-law behavior. However, for a strongly repulsive electron-electron interac- tion which suppresses the electron density of states near the quantum dot due to reflection of the conduction electrons, the local spin becomes nearly free, and the spin susceptibility shows simple Curie-type behavior.

In our analysis we shall assume that the quantum dot is embedded in an infinite quantum wire, the conduction electrons may tunnel from the quantum wire to the quantum dot, or vice versa. Notice that one may also consider another similar system with a quantum dot weakly connected to two half-infinite quantum wires with open boundary constraint conditions $^{12,13}$ at the quantum dot site. As shown in Refs. 14-18, in the former case and for electrons with repulsive interaction, the system goes to an infrared critical fixed point where the electrons are completely reflected from the quantum dot. In this limit the two quantum-dot systems should show similar low energy behavior of tunneling I-V. Notice, however that the low energy physics in these two cases are in general different. In the former case the complete reflection of electrons on the quantum dot is induced by the backward scattering potential which is relevant, while in the latter it is a boundary constraint and the backward scattering potential becomes irrelevant.

We shall describe our system by the following Hamiltonian,

$$
\begin{gathered}
H=H_{0}+H_{d}+H_{t} \\
H_{0}=-i \hbar \mathrm{v}_{F} \int d x\left[\psi_{R \sigma}^{\dagger}(x) \partial_{x} \psi_{R \sigma}(x)-\psi_{L \sigma}^{\dagger}(x) \partial_{x} \psi_{L \sigma}(x)\right] \\
+\frac{V}{2} \int d x \rho_{R \sigma}(x) \rho_{L \sigma}(x)+\frac{e V_{g}}{2} \sum_{\sigma} \int d x\left[\rho_{R \sigma}(x)\right. \\
\left.-\rho_{L \sigma}(x)\right] \\
H_{d}=\epsilon_{0} d_{\sigma}^{\dagger} d_{\sigma}+U d_{\uparrow}^{\dagger} d_{\uparrow} d_{\downarrow}^{\dagger} d_{\downarrow} \\
H_{t}=t_{0}\left\{d_{\sigma}^{\dagger}\left[\psi_{R \sigma}(0)+\psi_{L \sigma}(0)\right]+\left[\psi_{R \sigma}^{\dagger}(0)+\psi_{L \sigma}^{\dagger}(0)\right] d_{\sigma}\right\},
\end{gathered}
$$

where $\psi_{R \sigma}(x)$ are the right-moving electron fields, $\psi_{L \sigma}(x)$ are the left-moving electron fields, $\rho_{R(L) \sigma}(x)$ $=\psi_{R(L) \sigma}^{\dagger}(x) \psi_{R(L) \sigma}(x), V_{g}$ is an external voltage added between two ends of the quantum dot, $d_{\sigma}$ describes the electron in the level $\epsilon_{0}$ of the quantum dot, $U$ is the on site Coulomb repulsion, and $t_{0}$ is the hybridization amplitude between the 
quantum dot and wire. Here, we have assumed that the quantum dot is small enough to have well separated levels $\Delta \epsilon$ $=\epsilon_{n}-\epsilon_{n-1} \gg \Gamma$, and the dot can be seen as a structureless point at coordinate space. This model cannot be rigorously solved, and its physical behavior strongly depends on the parameters $\epsilon_{0}$ and $U$. In the low energy region, the tunneling $\mathrm{I}-\mathrm{V}$ of the system is mainly controlled by the occupation number of the level $\epsilon_{0}$ of the quantum dot (assuming other levels below it are occupied by even electrons and levels above are unoccupied). The Hilbert space of the local electron state $d_{\sigma}$ composes of four states $\{|0\rangle,|\uparrow\rangle,|\downarrow\rangle,|\uparrow \downarrow\rangle\}$, and its ground state depends on the value of $\epsilon_{0}$. In Sec. II, we shall consider the case with $\left\langle d_{\sigma}^{\dagger}(t) d_{\sigma}(t)>\cong 0\right.$, i.e., $\epsilon_{0} \gg \Gamma$, the level is far above the Fermi level of the quantum wire. In this limit, the quantum dot appears as a potential barrier to the conduction electrons. Using bosonization and phase shift representations, we show the evolution of the system from ultraviolet to infrared critical fixed points, and recover the known low energy physics at the infrared critical fixed point. In Sec. III, we study the case with $0 \ll d_{\sigma}^{\dagger}(t) d_{\sigma}(t)><1$, and $\left|\epsilon_{0}\right| \leqslant \Gamma$. In this case, the conduction electrons resonant with the electron level $d_{\sigma}$ in the quantum dot. In Sec. IV, we consider the case $<d_{\sigma}^{\dagger}(t) d_{\sigma}(t)>\cong 1$, i.e., $\epsilon_{0} \ll-\Gamma$, the level $\epsilon_{0}$ is far below the Fermi level of the quantum wire. In this case, the system is in the usual Kondo regime of Luttinger liquid. We shall show that all known previous results on this problem can be obtained in relatively straightforward ways using our approach. Finally, we give our conclusion and discussions in Sec. V where the advantage of our method will be pointed out.

\section{II. $\left(\epsilon_{0} \gg \Gamma\right)$ NON-MAGNETIC IMPURITY-LIKE SCATTERING IN THE NON-EQUILIBRIUM REGIME}

In the case of $\epsilon_{0} \gg \Gamma$, the level $\epsilon_{0}$ is unoccupied at equilibrium. The $U$-term in $H_{d}$ can be neglected, and we can simply integrate out the electron field $d_{\sigma}$ to obtain an effective Hamiltonian

$$
\begin{gathered}
H_{e f f}=H_{0}+H_{i m} \\
H_{i m}=U_{0}\left[\psi_{R \sigma}^{\dagger}(0) \psi_{R \sigma}(0)+\psi_{L \sigma}^{\dagger}(0) \psi_{L \sigma}(0)\right] \\
+U_{2 k_{F}}\left[\psi_{R \sigma}^{\dagger}(0) \psi_{L \sigma}(0)+\psi_{L \sigma}^{\dagger}(0) \psi_{R \sigma}(0)\right],
\end{gathered}
$$

where $U_{0} \sim U_{2 k_{F}} \sim t_{0}^{2} / \epsilon_{0} \sim \Gamma$. The $U_{0}$-term represents usual forward scattering which does not influence the transport behavior of the system, and can be neglected. The $U_{2 k_{F}}$-term describes usual backward scattering which determines the low energy behavior of the system, i.e., the system reduces to a single impurity scattering problem. In usual equilibrium renormalization group treatment with $V_{g}=0$, the backward scattering potential $U_{2 k_{F}}$ is a relevant quantity for repulsive electron-electron interaction $V>0$, and has a renormalized form $^{14} \quad U_{2 k_{F}}(l)=U_{2 k_{F}} e^{(1-g) l}, \quad$ where $\quad g=\left(2 \pi \hbar \mathrm{v}_{F}\right.$ $\left.-V / 2 \pi \hbar \mathrm{v}_{F}+V\right)^{1 / 2}$, and $l$ is the renormalization scale parameter. In the low energy limit $l \rightarrow \infty$, the renormalized backward scattering potential $U_{2 k_{F}}(l)$ goes to infinity and the low energy behavior of the system is determined by an effective (renormalized) Hamiltonian which can be obtained from (2) with the backward scattering potential $U_{2 k_{F}}$ replaced by the renormalized one $U_{2 k_{F}}(l)$. Due to the singular low energy behavior of the backward scattering potential, it is inconvenient to study the low energy behavior of the system with this effective Hamiltonian. One has to conjecture ${ }^{14}$ that at zero temperature where the backward scattering potential $U_{2 k_{F}}(l)$ goes to infinity, the electrons are completely reflected by the quantum dot at $x=0$. This conjecture can be justified with an alternative phase representation of the model $^{17,18}$ which we shall discuss in the following.

Here we consider this problem in the non-equilibrium case $^{19} V_{g} \neq 0$. For simplicity, we consider spinless fermions because at present the spin freedom appears only as a channel index. We first introduce a set of new electron fields

$$
\begin{aligned}
& \psi_{1}(x)=\frac{1}{\sqrt{2}}\left[\psi_{R}(x)+\psi_{L}(-x)\right], \\
& \psi_{2}(x)=\frac{1}{\sqrt{2}}\left[\psi_{R}(x)-\psi_{L}(-x)\right] .
\end{aligned}
$$

It is easy to check that the operators $\psi_{1(2)}(x)$ obey the standard anticommutation relations. The bosonic representation $^{20-22}$ of $\psi_{1(2)}(x)$ is $\psi_{1(2)}(x)=(D / 2 \pi)^{1 / 2} \exp$ $\left\{-i \Phi_{1(2)}(x)\right\}$, where $\partial_{x} \Phi_{1(2)}(x)=2 \pi \rho_{1(2)}(x)$. In terms of these new fields, the Hamiltonian (2) can be written as

$$
\begin{aligned}
H_{e f f}= & H_{0}+H_{i m} \\
H_{0}= & -i \int d x\left[\psi_{1}^{\dagger}(x) \partial_{x} \psi_{1}(x)+\psi_{2}^{\dagger}(x) \partial_{x} \psi_{2}(x)\right] \\
& +\frac{V}{4} \int d x\left\{\left[\rho_{1}(x)+\rho_{2}(x)\right]\left[\rho_{1}(-x)+\rho_{2}(-x)\right]\right. \\
& \left.-\left[\psi_{1}^{\dagger}(x) \psi_{2}(x)+\text { h.c. }\right]\left[\psi_{1}^{\dagger}(-x) \psi_{2}(-x)+\text { h.c. }\right]\right\} \\
& +\frac{e V_{g}}{2} \int d x\left[\psi_{1}^{\dagger}(x) \psi_{2}(x)+\psi_{2}^{\dagger}(x) \psi_{1}(x)\right] \\
& H_{i m}=U_{2 k_{F}}\left[\rho_{1}(0)-\rho_{2}(0)\right],
\end{aligned}
$$

where $\rho_{1(2)}(x)=\psi_{1(2)}^{\dagger}(x) \psi_{1(2)}(x)$. We have neglected the forward scattering term which does not influence the transport properties of the system.

In the phase shift representation, ${ }^{17,18}$ we obtain an effective renormalized Hamiltonian directly from (4)

$$
\begin{gathered}
H_{e f f}=H_{0}+H_{I M} \\
H_{I M}=\hbar \mathrm{\vee}_{F} \delta\left[\rho_{1}(0)-\rho_{2}(0)\right]
\end{gathered}
$$

which describes the low energy behavior of the system with original Hamiltonian (2) or (4), where the phase shift $\delta$ is a renormalized quantity, $\quad \delta=\arctan \left[e^{(1 / g-1) l} \tan \left(\delta_{0}\right)\right], \quad \delta_{0}$ $=U_{2 k_{F}} /\left(\hbar \mathrm{v}_{F}\right)$. In Ref. 18 we derive a renormalization group equation for $\delta$ for small electron-electron interaction $V /\left(2 \pi \hbar \mathrm{v}_{F}\right) \ll 1$, and the renormalized phase shift is $\delta$ $\sim \arctan \left[e^{\gamma l} \tan \left(\delta_{0}\right)\right]$, where $\gamma=V /\left(2 \pi \hbar \mathrm{v}_{F}\right)$. However, we have shown in Refs. 17 and 18 that the dimensionless coupling constant $g$ determines all correlation functions' expo- 
nents. We therefore replace $\gamma$ by $1 / g-1$ since $1 / g-1 \sim \gamma$ at small $\gamma$. At zero temperature and $V_{g}=0$, the phase shift $\delta$ take a critical value $\delta^{c}=\pi / 2$ at $l \rightarrow \infty$, which corresponds to the $U_{2 k_{F}}(l) \rightarrow \infty$ infrared critical point of the system. Notice that the backward scattering term $H_{I M}$ is finite at this infrared critical point in the phase shift representation. Therefore, the effective Hamiltonian (5) can be used safely to study the transport properties and evolution of correlation exponents of the system from high energy $(l=0)$ to low energy $(l \rightarrow \infty)$ regions.

It is convenient to introduce the unitary transformation $\bar{H}_{e f f}=\hat{U}^{\dagger}\left(H_{0}+H_{I M}\right) \hat{U}$, where

$$
\hat{U}=\exp \left\{i \frac{\delta}{2 \pi}\left[\Phi_{1}(0)-\Phi_{2}(0)\right]\right\},
$$

and $\partial_{x} \Phi_{1(2)}(x)=2 \pi \rho_{1(2)}(x)$. Together with the global gauge transformations

$$
\psi_{1(2)}(x)=\bar{\psi}_{1(2)}(x) e^{i \theta_{1(2)},} \quad \theta_{1}-\theta_{2}=\delta,
$$

we obtain the effective Hamiltonian $\bar{H}_{e f f}^{c}$ at the infrared critical point $\left(\delta^{c}=\pi / 2\right)^{17,18}$

$$
\begin{aligned}
\bar{H}_{e f f}^{c}= & -i \hbar \mathrm{v}_{F} \int d x\left[\bar{\psi}_{R}^{\dagger}(x) \partial_{x} \bar{\psi}_{R}(x)-\bar{\psi}_{L}^{\dagger}(x) \partial_{x} \bar{\psi}_{L}(x)\right] \\
& +\frac{V}{2} \int d x\left[\bar{\rho}_{R}(x) \bar{\rho}_{R}(-x)+\bar{\rho}_{L}(x) \bar{\rho}_{L}(-x)\right] \\
& -\frac{e V_{g}}{2} \int_{0}^{\infty} d x\left[\bar{\rho}_{R}(x)-\bar{\rho}_{R}(-x)+\bar{\rho}_{L}(x)-\bar{\rho}_{L}(-x)\right],
\end{aligned}
$$

where $\quad \bar{\psi}_{R}(x)=\left[\bar{\psi}_{1}(x)+\bar{\psi}_{2}(x)\right] / \sqrt{2}, \quad \bar{\psi}_{L}(-x)=\left[\bar{\psi}_{1}(x)\right.$ $\left.-\bar{\psi}_{2}(x)\right] / \sqrt{2} ; \quad \bar{\rho}_{R(L)}(x)=\bar{\psi}_{R(L)}^{\dagger}(x) \bar{\psi}_{R(L)}(x)$. This Hamiltonian $\bar{H}_{\text {eff }}^{c}$ completely determines the low energy physical behavior of the system. Note that at the infrared critical point $\delta^{c}=\pi / 2$, the effect of the backward scattering induced by the quantum dot is to alter the interaction among the conduction electrons, and to completely separate the right- and leftmoving electrons. It also alters the coupling to the external voltage due to reflection of the electrons from the quantum dot.

The reflection of the conduction electrons on the quantum dot can be seen clearly from the relations

$$
\begin{aligned}
& \hat{U}^{\dagger} \psi_{R}(x) \hat{U} \\
& \quad=e^{i \theta_{1}} e^{-i \delta / 2} \cdot \begin{cases}\cos (\delta) \bar{\psi}_{R}(x)+i \sin (\delta) \bar{\psi}_{L}(-x), & x>0 \\
\bar{\psi}_{R}(x), & x<0\end{cases} \\
& \hat{U}^{\dagger} \psi_{L}(x) \hat{U} \\
& \quad=e^{i \theta_{1}} e^{-i \delta / 2} \cdot \begin{cases}\bar{\psi}_{L}(x), & x>0 \\
\cos (\delta) \bar{\psi}_{L}(x)+i \sin (\delta) \bar{\psi}_{R}(-x), & x<0\end{cases}
\end{aligned}
$$

With these relations, we can obtain the reflection and transmission rates of the electrons, $\mathcal{R}$ and $\mathcal{T}$, respectively, where

$$
\mathcal{R}=\sin ^{2}(\delta), \quad \mathcal{T}=\cos ^{2}(\delta)
$$

We shall show in the following that the tunneling conductance is proportional to the transmission rate $\mathcal{T}$. At the infrared critical point $\delta^{c}=\pi / 2$, Eq. (9) shows that the right- and left-moving electrons are completely reflected on the quantum dot,

$$
\begin{aligned}
& \left.\hat{U}^{\dagger} \psi_{R}(x) \hat{U}\right|_{\delta=\delta^{c}}=e^{i \theta_{1}-i \pi / 4} \cdot \begin{cases}i \bar{\psi}_{L}(-x), & x>0 \\
\bar{\psi}_{R}(x), & x<0\end{cases} \\
& \left.\hat{U}^{\dagger} \psi_{L}(x) \hat{U}\right|_{\delta=\delta^{c}}=e^{i \theta_{1}-i \pi / 4} \cdot \begin{cases}\bar{\psi}_{L}(x), & x>0 \\
i \bar{\psi}_{R}(-x) . & x<0\end{cases}
\end{aligned}
$$

The external voltage term can be written in a more compact form by taking another transformation,

$$
\begin{aligned}
& \bar{\psi}_{R}(x) \rightarrow \begin{cases}e^{i e V_{g} x /\left(\hbar \mathrm{v}_{F}\right)} \bar{\psi}_{R}(x), & x>0 \\
\bar{\psi}_{R}(x), & x<0\end{cases} \\
& \bar{\psi}_{L}(x) \rightarrow \begin{cases}\bar{\psi}_{L}(x), & x>0 \\
e^{i e V_{g} x /\left(\hbar \mathrm{v}_{F}\right)} \bar{\psi}_{L}(x), & x<0\end{cases}
\end{aligned}
$$

With this the effective Hamiltonian (8) can be rewritten as

$$
\begin{aligned}
\bar{H}_{e f f}^{c}= & -i \hbar \mathrm{v}_{F} \int d x\left[\bar{\psi}_{R}^{\dagger}(x) \partial_{x} \bar{\psi}_{R}(x)-\bar{\psi}_{L}^{\dagger}(x) \partial_{x} \bar{\psi}_{L}(x)\right] \\
& +\frac{V}{2} \int d x\left[\bar{\rho}_{R}(x) \bar{\rho}_{R}(-x)+\bar{\rho}_{L}(x) \bar{\rho}_{L}(-x)\right] \\
& +\frac{e V_{g}}{2} \int d x\left[\bar{\rho}_{R}(x)-\bar{\rho}_{L}(x)\right] .
\end{aligned}
$$

To study low-energy transports we first employ the equations $e \partial_{t}\left[\rho_{R}(x, t)+\rho_{L}(x, t)\right]+\partial_{x} \hat{J}(x, t)=0$, and $\partial_{t} \rho_{R(L)}(x, t)$ $=i / \hbar\left[H_{e f f}, \rho_{R(L)}(x, t)\right]$ to obtain the current operator

$$
\hat{J}(x, t)=e \mathrm{v}_{F}(1-\gamma)\left[\rho_{R}(x, t)-\rho_{L}(x, t)\right],
$$

where $\gamma=V /\left(2 \pi \hbar \mathrm{v}_{F}\right)$. We have used the bosonized expression $^{20-22}$ of the Hamiltonian $H_{\text {eff }}$ to obtain the current operator $\hat{J}(x, t)$. The factor $\gamma$ presents the influence of the electron-electron interaction on the current. Under the unitary transformation $\hat{U}$, the current operator becomes 


$$
\begin{gathered}
\hat{J}(x, t)=\hat{U}^{\dagger} \hat{J}(x, t) \hat{U}=e \mathrm{v}_{F}(1-\gamma) \cdot \begin{cases}\alpha \bar{\rho}_{R}(x, t)+\beta \bar{\rho}_{L}(-x, t)-\bar{\rho}_{L}(x, t)-\hat{I}^{\prime}(x, t), & x>0 \\
\bar{\rho}_{R}(x, t)-\beta \bar{\rho}_{R}(-x, t)-\alpha \bar{\rho}_{L}(x, t)-\hat{I}^{\prime}(-x, t), & x<0\end{cases} \\
\hat{I}^{\prime}(x, t)=i \sin (2 \delta)\left[e^{i 2 e V_{g} /\left(\hbar \mathrm{v}_{F}\right)} \bar{\psi}_{L}^{\dagger}(-x, t) \bar{\psi}_{R}(x, t)-e^{-i 2 e V_{g} /\left(\hbar \mathrm{v}_{F}\right)} \bar{\psi}_{R}^{\dagger}(x, t) \bar{\psi}_{L}(-x, t)\right]
\end{gathered}
$$

where $\alpha=[1+\cos (2 \delta)] / 2$, and $\beta=[1-\cos (2 \delta)] / 2$. With this expression, it is straightforward to obtain the tunneling current

$$
\bar{I}=\langle\hat{J}(x, t)\rangle=\frac{g e^{2}}{2 \pi \hbar} V_{g} \mathcal{T},
$$

and with corresponding tunneling conductance, $G$ $=g e^{2} / 2 \pi \hbar \mathcal{T}$. At zero temperature and with small finite external voltage $V_{g}$ which is taken as a low energy cutoff factor, the renormalization parameter saturates at $l=$ $-\ln \left(e V_{g} / D\right)$, where $D$ is the conduction bandwidth, and the renormalized phase shift is $\delta=\arctan \left[\left(e V_{g} / D\right)^{1-1 / g} \tan \left(\delta_{0}\right)\right]$, therefore the transmission rate $\mathcal{T}$ of the conduction electrons is

$$
\mathcal{T}=\frac{\left(e V_{g}\right)^{2\left(\frac{1}{g}-1\right)}}{\left(e V_{g}\right)^{2\left(\frac{1}{g}-1\right)}+D^{2\left(\frac{1}{g}-1\right)} \tan ^{2}\left(\delta_{0}\right)} .
$$

In the case $\delta_{0}=0$ (no quantum dot), the tunneling current (15) restores the usual form of an infinite quantum wire. For $\delta_{0} \neq 0$, the tunneling current (15) is also consistent with results from previous calculations. ${ }^{13,14,23}$

The simple expression of the transmission rate is one of our central results, and is exact to leading order in $V_{g}$. For the special case of $g=1 / 2$, this model can be exactly solved, and the exact expression of the tunneling current is known. ${ }^{14}$ As expected, difference between the tunneling current (15) and the exact result appears only in higher order. The deviation comes from two sources, one is from the renormalized phase shift which is obtained by perturbation method in our analysis, and the other one comes from our approximation of using only the critical point Hamiltonian $\bar{H}_{e f f}^{c}$ to calculate the tunneling current. Notice that in Ref. 24, the authors calculated the tunneling current for a weak interaction ( $g$ $\sim 1$ ) electron system in Born approximation, and their result is consistent with the tunneling current (15) in the small $V$ limit $1 / g \sim 1+V /\left(2 \pi \hbar \mathrm{\vee}_{F}\right)$. In Ref. 25 the authors argued that the frequency dependence of conductance should include two terms: one is $c_{1} \omega^{2}$, and another one is $c_{2} \omega^{2 / g-2}$, where $\omega$ is frequency, $c_{1}$ and $c_{2}$ are constants. The $\omega^{2}$-term is not universal, and depends on an artificial finite cutoff, such as $\omega_{c}$ in Eq.(5) of Ref. 25. For a finite quantum wire, one can choose $c_{1} \sim \hbar \mathrm{v}_{F} q_{c}$ as a reasonable cutoff, where $q_{c}=2 \pi / L, L$ is the length of the system. For an infinite quantum wire, $c_{1}$ goes to zero and the $\omega^{2}$-term does not appear.

We have so far used only the effective Hamiltonian at the infrared critical point $\delta^{c}=\pi / 2$ to calculate the tunneling current. However, rigorously speaking, in the presence of small finite external voltage $V_{g}$, the system will deviate from the infrared critical point, and the low energy physical properties of the system would be determined by the Hamiltonian $H_{T}$ $=\bar{H}_{e f f}^{c}+\Delta H, \quad$ where $\quad \Delta H=-\hbar \mathrm{v}_{F}\left(\delta^{c}-\delta\right)\left[\bar{\psi}_{R}^{\dagger}(0) \bar{\psi}_{L}(0)\right.$ $\left.+\bar{\psi}_{L}^{\dagger}(0) \bar{\psi}_{R}(0)\right]$ is a small perturbation around the infrared critical point. The quantity $\delta^{c}-\delta \ll 1$ presents the deviation of the system away from the infrared critical point. We shall now show that the perturbative term $\Delta H$ only contributes to high order correction to the tunneling current. The corrections to tunneling current from $\Delta H$ can be estimated perturbatively. We obtain,

$$
\begin{aligned}
\bar{I}= & \langle\hat{\bar{J}}(x, t)\rangle-\frac{i}{L} \int d x \int d t^{\prime}\left\langle\hat{J}(x, t) \Delta H\left(t^{\prime}\right)\right\rangle \\
& -\frac{1}{L} \int d x \int d t^{\prime} d t^{\prime \prime}\left\langle\hat{\bar{J}}(x, t) \Delta H\left(t^{\prime}\right) \Delta H\left(t^{\prime \prime}\right)\right\rangle+\ldots
\end{aligned}
$$

where we have taken an average over space coordinate $x$, and $L \rightarrow \infty$ is the length of the system. The leading order correction comes from the operator $\hat{I}^{\prime}(x, t)$, and is proportional to $\left(e V_{g}\right)^{2(1 / g-1)}\left(\delta^{c}-\delta\right) \sin (2 \delta) / L$, where $\delta^{c}-\delta$ can be written as $\delta^{c}-\delta=\arcsin [\cos (\delta)]$. For small external voltage $V_{g}$, this correction can be neglected safely.

Before proceeding to next section, we first summarize our findings so far. Using the phase shift representation, we have calculated the tunneling current for the quantum-dot problem in the limit $\epsilon_{0} \gg \Gamma$, in which the problem can be mapped to the problem of non-magnetic impurity scattering. We obtain results in agreement with previous perturbation ${ }^{13,14}$ and Bethe ansatz $\mathrm{z}^{23}$ calculations. The phase representation method has the advantage that explicit divergence in the effective backward scattering amplitude is avoided, and the influence of the backward scattering on the system, such as the evolution of the Hamiltonian from high energy to low energy regions, the change of the effective interaction among the electrons, and the evolution of correlation exponents from ultraviolet $(\delta=0)$ to infrared $\left(\delta^{c}=\pi / 2\right)$ critical points, can be seen clearly. By introducing a simple unitary transformation on the original electron fields, we can obtain in a straightforward way the reflectivity and transmission rates of the electrons through the quantum dot, and then obtain the tunneling conductance of the system. It is one of the most prominent characteristics of one-dimensional interacting electron systems that the backward scattering of electrons on impurity (barrier) alters the effective electron-electron interaction because the right- and left-moving electrons are mixed by this scattering. This change in effective electron-electron interaction induces the observed singular low energy behaviors in the system. In the phase representation this line of physics is demonstrated clearly and naturally. 


\section{III. $\left(\epsilon_{0} \sim 0\right)$ ELECTRON RESONANT SCATTERING BY THE LOCAL LEVEL}

In the case $\epsilon_{0} \sim 0$, the level $\epsilon_{0}$ is close to the Fermi level of the quantum wire, and its electron occupation number satisfies $0 \ll d_{\sigma}^{\dagger}(t) d_{\sigma}(t)><1$. The resonance between the conduction electrons $\psi_{R(L) \sigma}(x)$ and the local electron $d_{\sigma}$ determines the low energy properties of the system. We shall treat the term $U d_{\uparrow}^{\dagger} d_{\uparrow} d_{\downarrow}^{\dagger} d_{\downarrow}$ by mean field theory in the following. In this approximation the $U$-term only renormalizes the energy ${ }^{26} \epsilon_{0}$ and the Hamiltonian (1) reduces to

$$
\begin{aligned}
H= & -i \hbar \mathrm{v}_{F} \int d x\left[\psi_{R \sigma}^{\dagger}(x) \partial_{x} \psi_{R \sigma}(x)-\psi_{L \sigma}^{\dagger}(x) \partial_{x} \psi_{L \sigma}(x)\right] \\
& +V \int d x \rho_{R \sigma}(x) \rho_{L \sigma}(x)+\frac{e V_{g}}{2} \sum_{\sigma} \int d x\left[\rho_{R \sigma}(x)\right. \\
& \left.-\rho_{L \sigma}(x)\right]+t_{0}\left\{\left[\psi_{R \sigma}^{\dagger}(0)+\psi_{L \sigma}^{\dagger}(0)\right] d_{\sigma}+d_{\sigma}^{\dagger}\left[\psi_{R \sigma}(0)\right.\right. \\
& \left.\left.+\psi_{L \sigma}(0)\right]\right\}+U_{2 k_{F}}\left[\psi_{R \sigma}^{\dagger}(0) \psi_{L \sigma}(0)+\psi_{L \sigma}^{\dagger}(0) \psi_{R \sigma}(0)\right] \\
& +\tilde{\epsilon}_{0} d_{\sigma}^{\dagger} d_{\sigma},
\end{aligned}
$$

where $\tilde{\epsilon}_{0}$ is the modified energy of the local electron $d_{\sigma}$. The Hamiltonian (18) is different from the previous one in Sec. II (2) by the presence of on site energy term for the local electron and the hybridization term between the conduction electrons and the local electron. The bare backward scattering potential $U_{2 k_{F}}$ is small because of strong resonance between the conduction electrons and the local electron orbital $d_{\sigma}$. However, the backward scattering term is relevant, no matter how small the bare backward scattering potential is, and will be renormalized to infinity in the low energy limit. The low energy behavior of the system is thus controlled by an effective Hamiltonian in which the renormalized backward scattering potential goes to infinity at the infrared critical point and the main transport comes from resonant tunneling. Notice that due to absence of spin exchange interactions in (18), the spin freedom appears only as a channel index. For simplicity, we shall only consider a spinless model with the same interaction form as the Hamiltonian (18). Using the same method as that in Sec. II, we obtain the effective Hamiltonian at the infrared critical point $\delta^{c}=\pi / 2$,

$$
\begin{aligned}
\bar{H}^{c}= & -i \hbar \mathrm{v}_{F} \int d x\left[\bar{\psi}_{R}^{\dagger}(x) \partial_{x} \bar{\psi}_{R}(x)-\bar{\psi}_{L}^{\dagger}(x) \partial_{x} \bar{\psi}_{L}(x)\right] \\
& +\frac{V}{2} \int d x\left[\bar{\rho}_{R}(x) \bar{\rho}_{R}(-x)+\bar{\rho}_{L}(x) \bar{\rho}_{L}(-x)\right] \\
& +\frac{e V_{g}}{2} \int d x\left[\bar{\rho}_{R}(x)-\bar{\rho}_{L}(x)\right]+t_{0}\left\{e ^ { - i \theta _ { 1 } + i \pi / 4 } \left[\bar{\psi}_{R}^{\dagger}(0)\right.\right. \\
& \left.\left.+\bar{\psi}_{L}^{\dagger}(0)\right] f+e^{i \theta_{1}-i \pi / 4} f^{\dagger}\left[\bar{\psi}_{R}(0)+\bar{\psi}_{L}(0)\right]\right\},
\end{aligned}
$$

where we have replaced $d_{\sigma}$ by $f$ for the spinless case.

Using the continuity equation, we obtain the current operator

$$
\hat{J}_{T}(x, t)=\hat{J}(x, t)+\hat{J}_{r}(t)
$$

$$
\begin{aligned}
\hat{J}_{r}(t)= & \frac{i e t_{0}}{\hbar}\left\{\left[\psi_{R}^{\dagger}(0, t)-\psi_{L}^{\dagger}(0, t)\right] f(t)-f^{\dagger}(t)\left[\psi_{R}(0, t)\right.\right. \\
& \left.\left.-\psi_{L}(0, t)\right]\right\},
\end{aligned}
$$

where $\hat{J}(x, t)$ is given by Eq. (13). $\hat{J}_{r}(t)$ is a resonant current operator induced by the resonant term in (18). Under the unitary transformation (6), the current operator becomes

$$
\begin{gathered}
\hat{\bar{J}}_{T}(x, t)=\hat{U}^{\dagger} \hat{J}_{T}(x, t) \hat{U}=\hat{\bar{J}}(x, t)+\hat{\bar{J}}_{r}(t) \\
\hat{\bar{J}}_{r}(t)=\frac{i e t_{0}}{\hbar}\left\{e^{-i \theta_{1}+i \pi / 4}\left[\bar{\psi}_{R}^{\dagger}(0, t)-\bar{\psi}_{L}^{\dagger}(0, t)\right] f(t)\right. \\
\left.-e^{i \theta_{1}-i \pi / 4} f^{\dagger}(t)\left[\bar{\psi}_{R}(0, t)-\bar{\psi}_{L}(0, t)\right]\right\},
\end{gathered}
$$

where the current operator $\hat{\bar{J}}(x, t)$ is given by Eq.(14).

Using the effective Hamiltonian (19) and the current operator (21), we can calculate the current of the system. The tunneling current $\langle\hat{\bar{J}}(x, t)\rangle$ induced by the backward scattering retains the same form as that in (15) because the resonant term in (19) only contributes a high order correction to it.

The resonant current $\left\langle\hat{\bar{J}}_{r}(t)\right\rangle$ originating from resonant tunneling between the conduction electrons and the local electron $f$ can be written as

$$
\bar{I}_{r}=\left\langle\hat{\bar{J}}_{r}(t)\right\rangle=\frac{e t_{0}}{\hbar}\left[G_{f \psi}(t, t)-G_{\psi f}(t, t)\right],
$$

where $\quad G_{f \psi}\left(t, t^{\prime}\right)=i\left\langle\left[\bar{\psi}_{R}^{\dagger}(0, t)-\bar{\psi}_{L}^{\dagger}(0, t)\right] f\left(t^{\prime}\right)\right\rangle e^{-i \theta_{1}+i \pi / 4}$, and $\quad G_{\psi f}\left(t, t^{\prime}\right)=i<f^{\dagger}(t)\left[\bar{\psi}_{R}\left(0, t^{\prime}\right)-\bar{\psi}_{L}\left(0, t^{\prime}\right)\right]>e^{i \theta_{1}-i \pi / 4}$. Applying usual perturbation method, we obtain for $G_{f \psi}\left(t, t^{\prime}\right)^{4,5,7}$

$$
\begin{aligned}
G_{f \psi}\left(t, t^{\prime}\right)= & t_{0} \int d t_{1}\left[G_{R L}\left(t, t_{1}\right) G_{f}^{a}\left(t_{1}, t^{\prime}\right)\right. \\
& \left.+G_{R L}^{r}\left(t, t_{1}\right) G_{f}\left(t_{1}, t^{\prime}\right)\right],
\end{aligned}
$$

where $\quad G_{R L}\left(t, t^{\prime}\right)=G_{R}\left(t, t^{\prime}\right)-G_{L}\left(t, t^{\prime}\right), \quad G_{R(L)}\left(t, t^{\prime}\right)$ $=i\left\langle\bar{\psi}_{R(L)}^{\dagger}(0, t) \bar{\psi}_{R(L)}\left(0, t^{\prime}\right)\right\rangle, G_{f}\left(t, t^{\prime}\right)=i\left\langle f^{\dagger}(t) f\left(t^{\prime}\right)\right\rangle$, and the up index $r$ and $a$ present the advanced and retarded Green functions, respectively. The Green functions satisfy the Dyson equations $\left(G_{f}^{a, r}\right)^{-1}=\left(G_{f}^{0, a, r}\right)^{-1}-\Sigma_{f}^{a, r}$, and $G_{f}$ $=G_{f}^{r} \Sigma_{f} G_{f}^{a}$, where $\Sigma_{f}^{r, a}=t_{0}^{2}\left[G_{R}^{r, a}+G_{L}^{r, a}\right]$ and $\Sigma_{f}=t_{0}^{2}\left[G_{R}\right.$ $\left.+G_{L}\right]$. In the low energy limit, the self-energy has the asymptotic behavior $\Sigma_{f}^{r, a}(\omega) \sim \omega^{-1+1 / g}$, and the electron Green functions can be written $\operatorname{as}^{27} \quad G_{R}(\omega) \sim n(\omega$ $\left.+e V_{g} / 2\right) \omega^{-1+1 / g}$, and $G_{L}(\omega) \sim n\left(\omega-e V_{g} / 2\right) \omega^{-1+1 / g}$, where $n\left(\omega \pm e V_{g} / 2\right)$ is the Fermi-Dirac distribution function. Therefore the Green function $G_{f}^{r, a}$ has low-energy asymptotic form $G_{f}^{r, a}(\omega) \sim 1 / \omega-\epsilon_{0}^{\prime}+i a \omega^{-1+1 / g}$, where $\epsilon_{0}^{\prime}$ is the renormalized energy of the local electron $f$ and $a$ is a constant $\sim t_{0}^{2}$. To leading order in $V_{g}$, the resonant current is given by

$$
\bar{I}_{r}=\frac{g e^{2}}{2 \pi \hbar} \frac{a^{2} V_{g}^{2 / g-1}}{\left(e V_{g}-\epsilon_{0}^{\prime}\right)^{2}+a^{2} V_{g}^{-2+2 / g}} .
$$


Notice that at $e V_{g}=\epsilon_{0}^{\prime}$, the resonant current becomes ${ }^{15} \bar{I}_{r}$ $=g e^{2} V_{g} /(2 \pi \hbar)$, with a linear relation between the current and the external voltage, similar to the case of free electron system. In the limit $\epsilon_{0}^{\prime} \gg e V_{g}$, we obtain ${ }^{15} \bar{I}_{r} \propto V_{g}^{2 / g-1} / \epsilon_{0}{ }_{0}^{2}$. In the former case, the current of the system is dominant by the resonant current. In the latter case, the resonant current only contributes a small correction to the current, which is consistent with our calculation in Sec. II where we consider the limit of large $\epsilon_{0}$ and the local electron field $d_{\sigma}$ is integrated out. The resonant current (24) provides an interpolation between these two regions which can be tested experimentally.

\section{IV. $\left(\epsilon_{0} \ll-\Gamma\right)$ QUANTUM SCATTERING OF A SPIN-1/2 MAGNETIC IMPURITY}

In the case of $\epsilon_{0} \ll-\Gamma$, the level $\epsilon_{0}$ is always occupied by an electron at equilibrium, $\left\langle d_{\sigma}^{\dagger}(t) d_{\sigma}(t)>\sim 1\right.$. In the large $U$ limit, and after making the Schrieffer-Woelf transformation, we obtain an effective Hamiltonian which describes the system in the Kondo regime,

$$
\begin{gathered}
H=H_{0}+H_{K} \\
H_{0}=-i \hbar \mathrm{\vee}_{F} \int d x\left[\psi_{R \sigma}^{\dagger}(x) \partial_{x} \psi_{R \sigma}(x)-\psi_{L \sigma}^{\dagger}(x) \partial_{x} \psi_{L \sigma}(x)\right] \\
+V \int d x \rho_{R \sigma}(x) \rho_{L \sigma}(x)+\frac{e V_{g}}{2} \sum_{\sigma} \int d x\left[\rho_{R \sigma}(x)\right. \\
\left.-\rho_{L \sigma}(x)\right] \\
H_{K}=\sum_{j} J_{1}^{j}\left[s_{R j}(0)+s_{L j}(0)\right] \cdot S_{j}+\sum_{j} J_{2}^{j}\left[s_{R L j}(0)\right. \\
\left.+s_{L R j}(0)\right] \cdot S_{j},
\end{gathered}
$$

where $\mathbf{S}=\frac{1}{2} d_{\alpha}^{\dagger} \boldsymbol{\sigma}_{\alpha \beta} d_{\beta}$ is a local spin operator,

$$
\begin{gathered}
\mathbf{s}_{R(L)}(0)=\frac{1}{2} \psi_{R(L) \alpha}^{\dagger}(0) \boldsymbol{\sigma}_{\alpha \beta} \psi_{R(L) \beta}(0), \\
\mathbf{S}_{R L(L R)}(0)=\frac{1}{2} \psi_{R(L) \alpha}^{\dagger}(0) \boldsymbol{\sigma}_{\alpha \beta} \psi_{L(R) \beta}(0),
\end{gathered}
$$

and $\left(J_{1}^{j}, J_{2}^{j}, j=x, y, z\right) \sim t_{0}^{2} / U$. The local spin operator satisfies $\left\langle S_{z}^{2}(t)\right\rangle=1 / 4$, and its Hilbert space consists of two states $\{|\uparrow>,| \downarrow>\}$. In abelian bosonization the term $J_{2}^{z}\left[s_{R L z}(0)\right.$ $\left.+s_{L R z}(0)\right] S_{z}$ in (25) acts as a backward scattering term which is relevant, whereas the term $\sum_{j} J_{1}^{j}\left[s_{R j}(0)\right.$ $\left.+s_{L j}(0)\right] \cdot S_{j}$ is a usual Kondo interaction term.

In terms of the electron fields $\psi_{1(2) \sigma}(x)=1 / \sqrt{2}\left[\psi_{R \sigma}(x)\right.$ $\left.\pm \psi_{L \sigma}(-x)\right]$, the interaction terms between the conduction electrons and the local spin can be written as

$$
\begin{gathered}
\sum_{j} J_{1}^{j}\left[s_{R j}(0)+s_{L j}(0)\right] \cdot S_{j}=\sum_{j} J_{1}^{j}\left[s_{1 j}(0)+s_{2 j}(0)\right] \cdot S_{j} \\
\sum_{j} J_{2}^{j}\left[s_{R L j}(0)+s_{L R j}(0)\right] \cdot S_{j}=\sum_{j} J_{2}^{j}\left[s_{1 j}(0)-s_{j 2}(0)\right] \cdot S_{j}
\end{gathered}
$$

where $\mathbf{s}_{1(2)}(0)=\psi_{1(2) \alpha}^{\dagger}(0) \boldsymbol{\sigma}_{\alpha \beta} \psi_{1(2) \beta}(0)$. We shall treat the system by abelian bosonization where the bare potential $J_{2}^{z}$ will be renormalized to infinity in the low energy region. It is convenient to replace the Hamiltonian (25) by a renormalized form obtained from usual renormalization group method,

$$
\begin{gathered}
H_{e f f}=H_{0}+H_{K}^{R} \\
H_{K}^{R}=\sum_{j=x, y} J_{1}^{j}\left[s_{1 j}(0)+s_{2 j}(0)\right] \cdot S_{j}+\sum_{j=x, y} J_{2}^{j}\left[s_{1 j}(0)\right. \\
\left.-s_{2 j}(0)\right] \cdot S_{j}+4 \hbar \widetilde{\mathrm{v}}_{F} \widetilde{\delta}\left[s_{1 z}(0)+s_{2 z}(0)\right] S_{z} \\
+4 \hbar \mathrm{v}_{F} \delta\left[s_{1 z}(0)-s_{2 z}(0)\right] S_{z},
\end{gathered}
$$

where $\tilde{\mathrm{v}}_{F}=\mathrm{v}_{F}\left(1-\gamma^{2}\right)^{1 / 2}$, the bare phase shift $\widetilde{\delta}_{0}$ $=J_{1}^{z} /\left(4 \hbar \tilde{\mathrm{v}}_{F}\right)$, and $\delta=\arctan \left[e^{(1 / g-1) l} \tan \left(J_{2}^{z} / 4 \hbar \mathrm{v}_{F}\right)\right]$, where $l$ is the renormalization scale parameter. The renormalization of the bare phase shift $\widetilde{\delta}_{0}$ depends on the electron interaction strength and the phase shift $\delta$. At the infrared critical point $\delta^{c}=\pi / 2$, the Hamiltonian of the system has a simple form [see (30)], $\bar{H}_{e f f}^{c}=\bar{H}_{0}^{c}+H_{K}^{c}$, where $H_{K}^{c}$ can be rewritten as,

$$
\begin{aligned}
H_{K}^{c}= & 4 \hbar \widetilde{\mathrm{v}}_{F} \widetilde{\delta}\left[s_{1 z}(0)+s_{2 z}(0)\right] S_{z}+\frac{D}{2 \pi \hbar \mathrm{v}_{F}}\left\{J _ { 2 } \left[e^{-i \Phi_{+s}(0)} S^{+}\right.\right. \\
& \left.+ \text {h.c. }]+J_{1}\left[e^{-i \Phi_{+s}(0)-i 2 \Phi_{-s}(0)} S^{+}+h . c .\right]\right\} .
\end{aligned}
$$

Using the result ${ }^{17}\left\langle e^{-i \Phi_{ \pm s}(0, t)} e^{i \Phi_{ \pm s}(0,0)}\right\rangle \sim t^{-1 / g}$, as $t \rightarrow \infty$, we can estimate the conformal dimension of the $\widetilde{\delta}$-term in $H_{K}^{c}$. For the weakly repulsive electron interaction, $1 / 2<g<1$, the $\widetilde{\delta}$-term has conformal dimensions $3-1 / g$, and is irrelevant, therefore the renormalized phase shift goes to zero in the low energy limit (Toulouse limit), $\widetilde{\delta} \rightarrow \widetilde{\delta}_{c}=0$. In the case of strongly repulsive electron interaction, $g \geqslant 1 / 2$, the local spin becomes nearly free, the $\widetilde{\delta}$-term has conformal dimension one, and is marginal, the renormalized phase shift therefore takes a finite value in the low energy limit, $\widetilde{\delta} \rightarrow\left|\widetilde{\delta}_{c}\right| \leqslant \pi / 2$. We shall take the renormalized phase shift $\widetilde{\delta}$ as a Toulouse parameter, and assume that $\widetilde{\delta} \rightarrow \widetilde{\delta}_{c}$ in the Toulouse limit. In the case of $V=0$ (no interaction among conduction electrons) and $J_{2}^{j}=0$, the system reduces to a usual two-channel Kondo problem, and in the Toulouse limit the phase shift takes the value $28-30 \widetilde{\delta}_{c}(V=0)=-\pi / 2$. Note that in the abelian bosonization treatment of the Kondo problem, the spin rotational symmetry is artificially broken. ${ }^{28-30}$ In particular, if an original exchange interaction has the spin rotational symmetry, the renormalized effective exchange interaction should retain this symmetry. However, in abelian bosonization one only renormalize the component $J_{1(2)}^{z}$, and the components $J_{1(2)}^{x, y}$ are treated as parameters which represent the hybridization between the conduction electrons and the local spin at the Toulouse limit $\widetilde{\delta}_{c}$. The parameters $J_{1(2)}^{x, y}$ should become small at the critical points $\delta^{c}$ and $\widetilde{\delta}_{c}$ because they are irrelevant. This artificial broken-symmetry originates from the bosonic representation of the electron fields, the $\mathrm{z}$-component of the spin can be presented as a linear term of a boson field, then it can be absorbed into the $(\mathrm{x}, \mathrm{y})$ component of the spin by simple unitary transformations. While under these unitary transformations the conformal di- 
mensions of the $J_{1(2)}^{x, y}$-term are reduced, and they become irrelevant at the critical points $\delta^{c}$ and $\widetilde{\delta}_{c}$. We believe that this process should incorporate the renormalization effect of the exchange interaction potentials $J_{1(2)}^{x, y, z}$ even though one only takes $\delta^{c}$ and $\widetilde{\delta}_{c}$ determined by the renormalized $J_{2}^{z}$ and $J_{1}^{z}$, respectively. It is well-known ${ }^{26,28,29}$ that the abelian bosonization correctly describes the low energy physical property of the Kondo problem as that of Bethe ansatz and conformal field theory treatments ${ }^{31,32}$ where the spin rotational symmetry is retained.

Now we derive the effective Hamiltonian at the infrared critical point $\delta^{c}=\pi / 2$ which determines the low energy physical behavior of the system. To this purpose, we define an unitary transformation operator which can be used to treat exactly the $\widetilde{\delta}$ and $\delta$ terms in (27)

$$
\hat{U}^{\prime}=\exp \left\{i \frac{2 g \widetilde{\delta}}{\pi} \Phi_{+s}(0) S_{z}+i \frac{2 \delta}{\pi} \Phi_{-s}(0) S_{z}\right\},
$$

where $\quad \Phi_{ \pm s}(0)=\frac{1}{2}\left\{\left[\Phi_{1 \uparrow}(0)-\Phi_{1 \downarrow}(0)\right] \pm\left[\Phi_{2 \uparrow}(0)\right.\right.$ $\left.\left.-\Phi_{2 \downarrow}(0)\right]\right\}, \quad \partial_{x} \Phi_{1(2) \sigma}(x)=2 \pi \rho_{1(2) \sigma}(x), \quad$ and $\quad \rho_{1(2) \sigma}(x)$ $=\psi_{1(2) \sigma}^{\dagger}(x) \psi_{1(2) \sigma}(x)$. Under the unitary transformation $\bar{H}_{e f f}^{c}=\hat{U}^{\prime \dagger} H_{e f f} \hat{U}^{\prime}$ and with the gauge transformation

$$
\begin{gathered}
\psi_{1 \sigma}(x)=\bar{\psi}_{1 \sigma}(x) e^{i \theta_{1}}, \quad \psi_{2 \sigma}(x)=\bar{\psi}_{2 \sigma}(x) e^{i \theta_{2}}, \\
\theta_{1}-\theta_{2}=2 \eta \delta S_{z},
\end{gathered}
$$

where $\eta=+1$ for the $\sigma=\uparrow$ and $\eta=-1$ for $\sigma=\downarrow$, the Hamiltonian (27) becomes at the infrared critical point $\delta^{c}$ $=\pi / 2$,

$$
\begin{gathered}
\bar{H}_{e f f}^{c}=\hat{U}^{\prime \dagger} H_{e f f} \hat{U}^{\prime}=\bar{H}_{0}^{c}+H_{K}^{c} \\
\bar{H}_{0}^{c}=-i \hbar \mathrm{v}_{F} \int d x\left[\bar{\psi}_{R \sigma}^{\dagger}(x) \partial_{x} \bar{\psi}_{R \sigma}(x)-\bar{\psi}_{L \sigma}^{\dagger}(x) \partial_{x} \bar{\psi}_{L \sigma}(x)\right] \\
+\frac{V}{2} \int d x\left[\bar{\rho}_{R \sigma}(x) \bar{\rho}_{R \sigma}(-x)+\bar{\rho}_{L \sigma}(x) \bar{\rho}_{L \sigma}(-x)\right] \\
+\frac{e V_{g}}{2} \sum_{\sigma} \int d x\left[\bar{\rho}_{R \sigma}(x)-\bar{\rho}_{L \sigma}(x)\right] \\
H_{K}^{c}=\frac{J_{2} D}{2 \pi \hbar \mathrm{v}_{F}}\left\{e^{-i\left(1+\frac{2 g \tilde{\delta}}{\pi}\right) \Phi_{+s}(0)} S^{+}+e^{i\left(1+\frac{2 g \tilde{\delta}}{\pi}\right) \Phi_{+s}(0)} S^{-}\right\} \\
+\frac{J_{1} D}{2 \pi \hbar \mathrm{v}_{F}}\left\{e^{-i\left(1+\frac{2 g \widetilde{\delta}}{\pi}\right) \Phi_{+s}(0)} e^{-i 2 \Phi_{-s}(0)} S^{+}\right. \\
\left.+e^{i\left(1+\frac{2 g \widetilde{\delta}}{\pi}\right) \Phi_{+s}(0)} e^{i 2 \Phi_{-s}(0)} S^{-}\right\},
\end{gathered}
$$

where $\quad \bar{\psi}_{R \sigma}(x)=\left[\bar{\psi}_{1 \sigma}(x)+\bar{\psi}_{2 \sigma}\right] / \sqrt{2}, \quad \bar{\psi}_{L \sigma}(-x)=\left[\bar{\psi}_{1 \sigma}(x)\right.$ $\left.-\bar{\psi}_{2 \sigma}(x)\right] / \sqrt{2}, \quad \bar{\rho}_{R(L) \sigma}(x)=\bar{\psi}_{R(L) \sigma}^{\dagger}(x) \bar{\psi}_{R(L) \sigma}(x), \quad$ and $S^{ \pm}$ $=S_{x} \pm i S_{y}$. Here we have taken the transformation (11) with spin indices included and $J_{1}+J_{2}=J_{1}^{x}=J_{1}^{y}$ as well as $J_{1}$ $-J_{2}=J_{2}^{x}=J_{2}^{y}$. The interaction Hamiltonian $H_{K}^{c}$ between the conduction electrons and the local spin of the quantum dot depends only on the boson fields $\Phi_{ \pm s}(0)$, and cannot be explicitly presented by the electron fields $\bar{\psi}_{R(L) \sigma}(x)$. The last term in $H_{K}^{c}$ has a high conformal dimension [4+(1 $\left.+2 g \widetilde{\delta} / \pi)^{2}\right) /(2 g)$, and can be neglected in lowest order approximation. Therefore, at the infrared critical point $\delta^{c}$ $=\pi / 2$, only the boson field $\Phi_{+s}(0)$ interact with the local spin of the quantum dot. For simplicity, we shall use a spinless fermion to represent the local spin, $S^{-}=f, S^{+}=f^{\dagger}$, and $S_{z}=f^{\dagger} f-1 / 2$, the Hamiltonian $H_{K}^{c}$ can be rewritten as

$$
H_{K}^{c}=K\left[\Psi^{\dagger}(0) f+f^{\dagger} \Psi(0)\right],
$$

where $K=J_{2}\left(D / 2 \pi \hbar \vee_{F}\right)^{1 / 2}$, and $\Psi(0)=\left(D / 2 \pi \hbar \vee_{F}\right)^{1 / 2} \exp$ $\left\{-i(1+2 g \widetilde{\delta} / \pi) \Phi_{+s}(0)\right\}$ is an anyon field which anticommutates with the fermion field $f$.

Using Dyson equation and $H_{K}^{c}$, we can obtain the Green function of the fermion $f, G_{f}^{-1}=G_{f 0}^{-1}-\Sigma_{f}$, where the selfenergy $\Sigma_{f}$ is given by $\Sigma_{f}(t)=K^{2} G_{\Psi}(t)$, where $G_{\Psi}$ is the Green function of the anyon field $\Psi(0)$. With the Hamiltonian $\bar{H}_{\text {eff }}^{c}$ (30), we obtain the correlation function, ${ }^{17}$ $\left\langle e^{i \Phi_{+s}(0, t)} e^{-i \Phi_{+s}(0,0)}\right\rangle \sim t^{-1 / g}$ for large $t$. Therefore, the anyon Green function has the asymptotic behavior, $G_{\Psi}(t)$ $\sim t^{-\left(1+2 g \widetilde{\delta}_{c} / \pi\right)^{2} / g}$, where $\widetilde{\delta}_{c}$ is the value of the Toulouse parameter $\widetilde{\delta}$ in the Toulouse limit, and the fermion Green function can be written in the low energy limit,

$$
G_{f}(\omega)=\frac{1}{\omega-i b \omega^{-1+\left(1+\frac{2 g \widetilde{\delta}_{c}}{\pi}\right)^{2} / g},}
$$

where $b$ is a constant proportional to $K^{2}$. Usually, the fixed point $\widetilde{\delta}=0$ is unstable due to the Kondo interaction between the conduction electrons and the local spin. There exists an infrared critical point (corresponding to the Toulouse limit) defined by the renormalized $J_{1}^{z R}$ or the Toulouse parameter $\widetilde{\delta}_{c}$, the Hamiltonian at this infrared critical point determines the low energy physical behavior of the system. In the case of $V=0$ and $J_{2}^{j}=0$, the system reduces to a usual twochannel Kondo problem, and has an infrared critical point corresponding to $\widetilde{\delta}_{c}=-\pi / 2$. In the presence of the electronelectron interaction and $J_{2}^{j} \neq 0$, we assume the Toulouse parameter $\widetilde{\delta}_{c}$ satisfies $^{28-30}, 0 \geqslant \widetilde{\delta}_{c} \geqslant-\pi / 2$. In the case of weak repulsive electron-electron interaction $\kappa=\left(1+2 g \widetilde{\delta}_{c} / \pi\right)^{2} / g$ $<2$, the fermion Green function has the asymptotic behavior, $G_{f}(t) \sim t^{-2+\kappa}$ as $t \rightarrow \infty$. For the strong repulsive electronelectron interaction, i.e., $\kappa \gg 2$, the fermion Green function $G_{f}$ is a free-fermion Green's function at low energy, i.e., the local spin becomes free, which is consistent with our previous calculation. ${ }^{17,33}$

Now we consider the transmission and reflection of conduction electrons on the local spin of the quantum dot. Under the unitary transformation $\hat{U}^{\prime}$, the electron fields $\psi_{R(L) \sigma}(x)$ become

$$
\begin{aligned}
\hat{U}^{\prime} \psi_{R \sigma}(x) \hat{U}^{\prime}= & \frac{1}{2} e^{i \eta(g \tilde{\delta}+\delta) S_{z} \operatorname{sgn}(x)+i \theta_{1}} \\
& \times\left\{\left[1+e^{-i 2 \eta \delta S_{z}(1+\operatorname{sgn}(x))}\right] \bar{\psi}_{R \sigma}^{\prime}(x)\right. \\
& \left.+\left[1-e^{-i 2 \eta \delta S_{z}(1+\operatorname{sgn}(x))}\right] \bar{\psi}_{L \sigma}^{\prime}(-x)\right\}
\end{aligned}
$$




$$
\begin{aligned}
\hat{U}^{\prime} \psi_{L \sigma}(x) \hat{U}^{\prime}= & \frac{1}{2} e^{-i \eta(g \widetilde{\delta}+\delta) S_{z} \operatorname{sgn}(x)+i \theta_{1}} \\
& \times\left\{\left[1-e^{-i 2 \eta \delta S_{z}(1-\operatorname{sgn}(x))}\right] \bar{\psi}_{R \sigma}^{\prime}(-x)\right. \\
& \left.+\left[1+e^{-i 2 \eta \delta S_{z}(1-\operatorname{sgn}(x))}\right] \bar{\psi}_{L \sigma}^{\prime}(x)\right\},
\end{aligned}
$$

where $\quad \bar{\psi}_{R(L) \sigma}^{\prime}(x)=\exp \left\{i e V_{g} x \theta( \pm x) /\left(\hbar \mathrm{v}_{F}\right)\right\} \bar{\psi}_{R(L) \sigma}(x)$, and $\theta(x)=1$ for $x>0$ and $\theta(x)=0$ for $x<0$. According to these transformations, we can obtain the reflection and transmission rates of the electrons, $\mathcal{R}=\sin ^{2}(\delta)$, and $\mathcal{T}=\cos ^{2}(\delta)$, respectively, which is independent of the Toulouse parameter $\widetilde{\delta}$, and is the same as that of the barrier scattering (10). At the infrared critical point $\delta^{c}=\pi / 2$, the electron transmission rate is zero, and the electrons are completely reflected on the quantum dot. This result can be easily understood because in the abelian bosonization the exchange interaction term $J_{2}^{z}\left[s_{R L z}(0)+s_{L R z}(0)\right] S_{z}$ just provides a backward scattering of the conduction electrons on the local spin.

With the continuity equation $e \partial_{t} \Sigma_{\sigma}\left[\rho_{R \sigma}(x, t)\right.$ $\left.+\rho_{L \sigma}(x, t)\right]+\partial_{x} \hat{J}(x, t)=0$, we can derive the current operator

$$
\hat{J}(x, t)=e \mathrm{v}_{F}(1-\gamma) \sum_{\sigma}\left[\rho_{R \sigma}(x, t)-\rho_{L \sigma}(x, t)\right]
$$

which is the same as the current operator (13) in the case of barrier scattering $\epsilon_{0} \gg 0$, except the presence of extra spin indices. Under the unitary transformation $\hat{U}^{\prime}$, the density operators $\rho_{R \sigma}(x)$ become

$$
\begin{aligned}
\hat{U}^{\prime \dagger} \rho_{R \sigma}(x) \hat{U}^{\prime}= & \frac{1}{2}\left\{\bar{\rho}_{R \sigma}(x)+\bar{\rho}_{L \sigma}(-x)+\cos [\delta(1+\operatorname{sgn}(x))]\right. \\
& \times\left[\bar{\rho}_{R \sigma}(x)-\bar{\rho}_{L \sigma}(-x)\right]-i 2 \eta \\
& \times \sin [\delta(1+\operatorname{sgn}(x))] S_{z}\left[\bar{\psi}_{L \sigma}^{\prime \dagger}(-x) \bar{\psi}_{R \sigma}^{\prime}(x)\right. \\
& \left.\left.-\bar{\psi}_{R \sigma}^{\prime \dagger}(x) \bar{\psi}_{L \sigma}^{\prime}(-x)\right]\right\} \\
\hat{U}^{\prime \dagger} \rho_{L \sigma}(x) \hat{U}^{\prime}= & \frac{1}{2}\left\{\bar{\rho}_{R \sigma}(-x)+\bar{\rho}_{L \sigma}(x)-\cos [\delta(1-\operatorname{sgn}(x))]\right. \\
& \times\left[\bar{\rho}_{R \sigma}(-x)-\bar{\rho}_{L \sigma}(x)\right]+i 2 \eta \\
& \times \sin \left[\delta\left(1-\sin _{n}(x)\right)\right] S_{z}\left[\bar{\psi}_{L \sigma}^{\prime \dagger}(x) \bar{\psi}_{R \sigma}^{\prime}(-x)\right. \\
& \left.\left.-\bar{\psi}_{R \sigma}^{\prime \dagger}(-x) \bar{\psi}_{L \sigma}^{\prime}(x)\right]\right\}
\end{aligned}
$$

and the current operator $\hat{\mathcal{J}}(x, t)=\hat{U}^{\prime \dagger} \hat{J}(x, t) \hat{U}^{\prime}$ is $(x>0)$

$$
\begin{aligned}
\hat{\mathcal{J}}(x, t)= & e \mathrm{v}_{F}(1-\gamma) \sum_{\sigma}\left[\alpha \bar{\rho}_{R \sigma}(x, t)+\beta \bar{\rho}_{L \sigma}(-x, t)\right. \\
& \left.-\bar{\rho}_{L \sigma}(x, t)\right]-i 2 e \mathrm{v}_{F}(1-\gamma) \sin (2 \delta)\left[s_{L R z}(-x, x)\right. \\
& \left.-s_{R L z}(x,-x)\right] S_{z}
\end{aligned}
$$

where $s_{R L(L R) z}\left(x, x^{\prime}\right)=\frac{1}{2} \bar{\psi}_{R(L) \alpha}^{\prime \dagger}(x) \sigma_{\alpha \beta}^{z} \bar{\psi}_{L(R) \beta}^{\prime}\left(x^{\prime}\right)$ are nonlocal spin operators. Using the Hamiltonian $\bar{H}_{\text {eff }}^{c}$ (30), we obtain the current of the system

$$
\mathcal{I}=\langle\hat{\mathcal{J}}(x, t)\rangle=\frac{g e^{2}}{\pi \hbar} V_{g} \mathcal{T},
$$

where the electron transmission rate $\mathcal{T}$ is given by Eq.(16) with the bare $\delta_{0}$ replaced by $J^{z} /\left(4 \hbar \mathrm{v}_{F}\right)$. The last term in (36) does not contribute to the current, because at the infrared critical point $\delta^{c}=\pi / 2$ the right- and left-moving electrons are completely separated, so the average of the nonlocal spin operator is zero, $\left\langle s_{R L(L R) z}\left(x, x^{\prime}\right)\right\rangle=0$.

Note that as in previous cases, to study the low energy properties of the system with $V_{g} \neq 0$, we need to add a perturbative term $\Delta H=-4 \hbar \mathrm{v}_{F}\left(\delta^{c}-\delta\right)\left[s_{R L z}(0)+s_{L R z}(0)\right] S_{z}$ to the critical Hamiltonian $\bar{H}_{\text {eff }}^{c}(30)$, where $\Delta \delta=\delta^{c}-\delta$ $=\arcsin [\cos (\delta)] \ll 1$ presents the deviation of the system away from the infrared critical point $\delta^{c}=\pi / 2$. The first order correction of the current from the perturbation $\Delta H$ is $-4 e \mathrm{v}_{F}(1-\gamma) \sin (2 \delta) \int_{0}^{L / 2} d x \int d t^{\prime}\left\langle\left[s_{L R z}(-x, x, t)-s_{R L z}(x\right.\right.$, $\left.-x, t)] S_{z}(t) \Delta H\left(t^{\prime}\right)\right\rangle / L$, which goes to zero in the infinite quantum wire limit $L \rightarrow \infty$, and is proportional to $V_{g}^{4 / g-3}$ for the finite quantum wire $L \ll \hbar \mathrm{v}_{F} /\left(e V_{g}\right)$. Therefore, the correction of the current by the $\Delta H$ can be neglected, and the current of the system is presented by $\mathcal{I}(37)$ to lowest order in $V_{g}$. This result means that the tunneling current through the quantum dot has the same low energy behavior for both the cases of $\epsilon_{0} \gg \Gamma \quad\left[\left\langle d_{\sigma}^{\dagger}(t) d_{\sigma}(t)\right\rangle \sim 0\right]$ and $\epsilon_{0} \ll \Gamma$ $\left[\left\langle d_{\sigma}^{\dagger}(t) d_{\sigma}(t)\right\rangle \sim 1\right]$. However, in the latter case the spin susceptibility of the quantum dot is not zero.

The tunneling current (37) is one of our central results. This result is consistent with that of Ref. 13 where the authors used open boundary condition, and the magnetic impurity is residing at the end of a half-infinite quantum wire. Although similar expression of the low energy tunneling current is obtained in both cases, the microscopic physics appears to be rather different. At present case, the backwardscattering-like Kondo interaction $J_{2}^{i}\left[s_{R L i}(0)+s_{L R i}(0)\right] \cdot S_{i}$ is relevant for repulsive electron-electron interaction, and induces the complete reflection of electrons on the quantum dot at the critical fixed point $\delta^{c}=\pi / 2$. This relevant interaction also affects the usual Kondo interaction term $J_{1}^{i}\left[s_{R i}(0)+s_{L i}(0)\right] \cdot S_{i}$. On the other hand, in the open boundary case the complete reflection of electrons on the magnetic impurity is a boundary constraint. The backwardscattering-like Kondo interaction term is irrelevant, and contributes only a high order correction to the usual Kondo interaction. As far as electronic transport is concerned, the tunneling current of the system is determined only by the infrared critical fixed point ( $\delta^{c}=\pi / 2$ ) Hamiltonian, therefore similar tunneling current expression are obtained in these two cases. However, the impurity susceptibility is determined by both the infrared critical fixed point Hamiltonian as well as the coupling between the magnetic impurity and the conduction electrons at (or near) the infrared critical point. At present case, the usual two-channel Kondo fixed point is unstable due to the backward-scattering-like Kondo interaction, while in the open boundary case, the two-channel 
Kondo fixed point is stable for strong repulsive electronelectron interaction in which the backward-scattering-like Kondo interaction term is irrelevant. Therefore, the impurity susceptibility shows different low energy behavior in these two cases. $13,17,33$

\section{DISCUSSION AND CONCLUSION}

It will be possible in future experiments to fabricate and study a system consisting of a quantum dot or an artificial atom connected with an infinite quantum wire. The quantum dot or artificial atom can be made very small by modulating gate voltages, so that it has well-defined discrete energy levels. If the gap between two nearest levels is large compared with the hybridization $\Gamma$ between the dot and the quantum wire, the low energy physical behavior of the system is controlled by the level $\epsilon_{0}$ closest to the Fermi level of the quantum wire. With this simple system, one can experimentally study the physical property of an Anderson model with and without the electron-electron interaction in the mixed valence and single occupied states.

This system can be presented by an single-impurity Anderson model with electron-electron interaction, if we assume that the other levels below the level $\epsilon_{0}$ are occupied by even electrons, and above it are unoccupied. However, this simple model cannot be exactly solved and the nature of the ground state depends strongly on $\epsilon_{0}$. The Hilbert space of the local electron consists of $\{|0\rangle,|\uparrow\rangle,|\downarrow\rangle,|\uparrow \downarrow\rangle\}$. In the case of $\epsilon_{0}>0$, the ground state of the local electron is essentially just $|0\rangle .\left\langle d_{\sigma}^{\dagger}(t) d_{\sigma}(t\rangle\right) \sim 0$, and the quantum dot problem reduces to the problem of simple barrier-like potential scattering for conduction electrons. In the case of $\epsilon_{0} \sim 0$, the local electron is in a mixed valence state with $\left\langle d_{\sigma}^{\dagger}(t) d_{\sigma}(t)\right\rangle<1$, the local electron orbital is in resonance with the conduction electrons. In the case of $\epsilon_{0} \ll-\Gamma$, the system is in the Kondo regime. The local electron state is singly occupied state with $\left\langle d_{\sigma}^{\dagger}(t) d_{\sigma}(t)\right\rangle=1$, and the local electron has a Kondo exchange interaction with the conduction electrons, i.e., the system reduces to a one-dimensional Kondo problem with electron-electron interaction. In these three cases, the local electron orbital has very different states, and the system has very different low energy behavior.

With the bosonization method and phase shift representation, we studied in this paper the low energy transport be- haviors of the system in these three limit cases at zero temperature and with a small external voltage $V_{g}$. We have demonstrated that the current of the system has the same low energy power-law behavior $\sim V_{g}^{2 / g-1}$ for both cases of $\epsilon_{0}$ $\gg \Gamma$ and $\epsilon_{0} \ll-\Gamma$, even though the local electrons have completely different effects on conduction electrons in these two cases. In the former case, the local electron orbital only provides a barrier-like potential scattering, whereas in the latter, the local electron has the Kondo-type exchange interaction with the conduction electrons. The same low energy current expression originates from the fact that there exist similar backward scattering potentials in both cases, $U_{2 k_{F}}\left[\psi_{R \sigma}^{\dagger}(0) \psi_{L \sigma}(0)+\psi_{L \sigma}^{\dagger}(0) \psi_{R \sigma}(0)\right], \quad$ and $\quad J_{2}^{z}\left[s_{R L}(0)\right.$ $\left.+s_{L R}(0)\right] S_{z}$, respectively. Note that in general the low energy properties of the system are different in these two limit cases, such as the spin susceptibility of the local electron. Generally, in the case of $\epsilon_{0} \ll-\Gamma$, the local electron spin susceptibility shows power-law behavior at low energy, but for strong enough repulsive electron-electron interaction, the local spin becomes nearly free. This prominent property of one-dimensional Kondo problem originates from the almost complete reflection of the electrons on the local spin in the low energy limit. As a result the electron density of states near the local spin becomes small, and the magnitude of the spin exchange terms $s_{R(L)}^{+}(0) S^{-}$and $s_{R(L)}^{-}(0) S^{+}$are strongly reduced. In the case of $\epsilon_{0} \sim 0$, the local electron is in the mixed valence state. The system shows different low energy behavior from that of the above two cases. we demonstrate that even though the electrons are almost completely reflected by the local electron in the low energy region, the resonance between the local and conduction electrons enhances the tunneling current through the quantum dot. In this resonance case, the system has the same low energy transport properties as that of a simple model of a resonant level coupling to two half-infinite quantum wires where electron fields satisfy open boundary conditions at the resonant level site, and the tunneling current is proportional to $V_{g}$. The advantage of the phase shift representation is that by using the simple unitary transformation we can directly obtain the tunneling current without calculating correlation functions.

We acknowledge support of HKRGC through Grant No. UST6143/97P.
${ }^{1}$ T. K. Ng and P. A. Lee, Phys. Rev. Lett. 61, 1768 (1988).

${ }^{2}$ L. I. Glazman and M. E. Raikh, JETP Lett. 47, 452 (1988).

${ }^{3}$ A. Kawabata, J. Phys. Soc. Jpn. 60, 3222 (1991).

${ }^{4}$ Y. Meir and N. S. Wingreen, Phys. Rev. Lett. 68, 2512 (1992); Y.

Meir, N. S. Wingreen, and P. A. Lee, Phys. Rev. Lett. 70, 2601 (1993).

${ }^{5}$ S. Hershfield, J. H. Davies, and J. W. Wilkins, Phys. Rev. Lett. 67, 3720 (1991).

${ }^{6}$ A. L. Yeyati et al., Phys. Rev. Lett. 71, 2991 (1993).

${ }^{7}$ N. S. Wingreen and Y. Meir, Phys. Rev. B 49, 11040 (1994).

${ }^{8}$ Y. Wan et al., Phys. Rev. B 51, 14782 (1995).

${ }^{9}$ K. A. Matveev, Phys. Rev. B 51, 1743 (1995); I. L. Aleiner and L. I. Glazman, Phys. Rev. B 57, 9608 (1998).
${ }^{10}$ D. Goldhaber-Gordon et al., Nature (London) 391, 156 (1998).

${ }^{11}$ S. M. Cronenwett et al., Science 281, 540 (1998).

${ }^{12}$ S. Eggert and I. Affleck, Phys. Rev. B 46, 10866 (1992).

${ }^{13}$ M. Fabrizio and A. O. Gogolin, Phys. Rev. B 51, 17827 (1995).

${ }^{14}$ C. L. Kane and M. P. A. Fisher, Phys. Rev. Lett. 68, 1220 (1992); Phys. Rev. B 46, 15233 (1992).

${ }^{15}$ K. Moon, H. Yi, C. L. Kane, S. M. Girvin, and M. P. A. Fisher, Phys. Rev. Lett. 71, 4381 (1993); C. L. Kane and M. P. A. Fisher, Phys. Rev. B 52, 17393 (1995).

${ }^{16}$ A. O. Gogolin, Phys. Rev. Lett. 71, 2995 (1993); N. V. Prokof'ev, Phys. Rev. B 49, 2148 (1994); C. L. Kane, K. A. Matveev, and L. I. Glazman, Phys. Rev. B 49, 2253 (1994); I. Affleck and A. W. W. Ludwig, J. Phys. A 27, 5375 (1994); M. 
Fabrizio and A. O. Gogolin, Phys. Rev. Lett. 78, 4527 (1997); Y. Oreg and A. M. Finkel'stein, Phys. Rev. Lett. 78, 4528 (1997)

${ }^{17}$ Y. L. Liu, Phys. Rev. B 57, 12104 (1998); Phys. Rev. B 59, 7937 (1999).

${ }^{18}$ Y. L. Liu, Influence of Backward Scattering on Correlation Exponents in One-dimensional System, preprint.

${ }^{19}$ C. L. Kane and M. P. A. Fisher, Phys. Rev. Lett. 72, 724 (1994).

${ }^{20}$ A. Luther and I. Peschel, Phys. Rev. B 9, 2911 (1974).

${ }^{21}$ V. J. Emery, in Highly Conducting One-Dimensional Solids, edited by J. T. Devreese et al. (Plenum Press, New York, 1979); J. Sólyom, ADV. Phys. 28, 201 (1979).

${ }^{22}$ F. D. M. Haldane, J. Phys. C 14, 2585 (1981).

${ }^{23}$ P. Fendley, A. W. W. Ludwig, and H. Saleur, Phys. Rev. Lett. 74, 3005 (1995); F. Lesage, H. Saleur, and S. Skorik, ibid., 76, 3388 (1996).

${ }^{24}$ D. X. Yue, L. I. Glazman, and K. A. Matveev, Phys. Rev. B 49,
1966 (1994).

${ }^{25}$ F. Guinea, G. Gomez Santos, M. Sassetti, and M. Ueda, Europhys. Lett. 30, 561 (1995).

${ }^{26}$ A. C. Hewson, The Kondo Problem to Heavy Fermions (Cambridge University Press, Cambridge, 1993).

${ }^{27}$ A.P. Jauho, N. S. Wingreen, and Y. Meir, Phys. Rev. B 50, 5528 (1994).

${ }^{28}$ M. Fabrizio, A. O. Gogolin, and P. Nozières, Phys. Rev. B 51, 16 088 (1995).

${ }^{29}$ V. J. Emery and S. Kivelson, Phys. Rev. B 46, 10812 (1992).

${ }^{30}$ D. H. Lee and J. Toner, Phys. Rev. Lett. 69, 3378 (1992); A. Furusaki and N. Nagaosa, Phys. Rev. Lett. 72, 892 (1994); P. Frojdh and H. Johannesson, Phys. Rev. Lett. 75, 300 (1995).

${ }^{31}$ N. Andrei and C. Destri, Phys. Rev. Lett. 52, 364 (1984); A. M. Tsvelick and P. W. Wiegmann, Z. Phys. A 54, 201 (1984). ${ }^{32}$ I. Affleck and A. Ludwig, Nucl. Phys. B B360, 641 (1991).

${ }^{33}$ Y. L. Liu, Phys. Rev. Lett. 79, 293 (1997). 